\title{
Obtención de silicatos de calcio empleando como precursores residuos sólidos. Influencia del mezclado de reactivos en fase seca $o$ fase húmeda
}

\author{
M. FELIPE-SESÉ, D. ELICHE-QUESADA y F. A. CORPAS-IGLESIAS \\ Departamento de Ingeniería Química, Ambiental y de los Materiales. EPS de Linares, Universidad de Jaén, 23700 Linares (Jaén), Spain; \\ e-mail: facorpas@ujaen.es
}

\begin{abstract}
Se ha determinado la posibilidad de reutilizar los residuos procedentes del corte de mármol, como fuente de óxido de calcio, y las cenizas resultantes de la combustión de los residuos generados en el proceso de fabricación de tableros de derivados de madera, como fuente de sílice. A partir de ambas materias primas, se obtienen silicatos de calcio. Se ha estudiado la influencia de agua en la fase inicial de mezclado de los residuos. Para ello mármol y cenizas se han mezclado en relación molar $\mathrm{CaO}_{\mathrm{SiO}}$ 1:1, en una primera ruta, en fase seca, empleando un molino de bolas, o en una segunda ruta, en fase húmeda, mediante agitación a $90{ }^{\circ} \mathrm{C}(2 \mathrm{~h})$ empleando un $60 \%$ en peso de agua. Posteriormente, ambas mezclas se sinterizan a $1100{ }^{\circ} \mathrm{C}(24 \mathrm{~h})$. Con objeto de emplear los silicatos cálcicos obtenidos como materiales cerámicos aislantes térmicos de baja temperatura, se comprimen a $15 \mathrm{Tm}$ obteniendo ladrillos cuyas propiedades tecnológicas han sido estudiadas. Tanto los materiales cerámicos obtenidos por mezclado de los residuos en fase seca, como en fase húmeda, se pueden emplear como aislantes térmicos, presentando valores de conductividad de 0.18 y $0.12 \mathrm{w} / \mathrm{m}^{2} \mathrm{~K}$ respectivamente, con resistencia a la compresión elevada.
\end{abstract}

Palabras claves: silicatos de calcio, residuos de mármol y cenizas, reciclado.

Obtaining calcium silicates by using solid residues as precursors. Influence of water in the process of mixing reagents.

The suitability of re-using residues marble, remaining from cutting marble, as a source of calcium-oxide, as well as the resultant ashes from the combustion of the wastes generated in the process of manufacturing boards from derivates of wood, as a source of silica, as raw material for the production of calcium silicate products has been determined. First of all, the influence of water has been studied in the initial phase of mixing residues. Marble and ashes have been mixed in molar relation $\mathrm{CaO}: \mathrm{SiO}_{2}$ of $1: 1$ using two different ways: using a planetary ball mill (while in solid state) or agitating at $90{ }^{\circ} \mathrm{C}(2$ h) using a $60 \mathrm{wt} \%$ of water (while in humid state). Later, both mixtures were sintered at $1100{ }^{\circ} \mathrm{C}(24 \mathrm{~h})$. In order to use the obtained calcium-silicates as ceramic insulating thermal materials, the samples were compressed at 15 Tm obtaining bricks from which the technological properties have been studied. The ceramic materials obtained from mixing the residues in dry phase, as well as those obtained in the wet phase, can be used as thermal insulators, showing values of conductivity of 0.18 and $0.12 \mathrm{w} / \mathrm{m}^{2} \mathrm{~K}$, with an elevated resistance to compressive strength.

Key words: Calcium silicates, marble and ash wastes, recycling.

\section{INTRODUCCIÓN}

En la actualidad, la industria a nivel mundial es responsable de la generación de grandes cantidades de residuos sólidos [1-5], de ahí que los cambios legislativos en materia medioambiental acontecidos en España, impulsados desde su ingreso en la Unión Europea, han conducido a la necesidad de dar respuesta al problema de la gestión de estos residuos procedentes de los diferentes procesos industriales.

En la industria del mármol, se generan residuos debido a la acumulación de grandes cantidades de polvo y lodo blanco durante el proceso de corte y pulido del material, que están constituidos principalmente por carbonato cálcico. Teniendo en cuenta que España es el segundo productor de mármol del mundo, y que el 95\% del mármol que se comercializa en España procede de la comarca de Macael (Almería), no es de extrañar que en el año 2001 se extrajeran de esta piedra un total de 2.232.223 toneladas, y teniendo en cuenta, que tan sólo en la operación de aserrado por cada $3000 \mathrm{~m}^{2}$ de mármol, se generan $600 \mathrm{Tm}$ de residuos, muestra las dimensiones de la situación.

En la industria de la fabricación de tableros derivados de la madera, se emplean cómo combustible para la obtención de energía eléctrica aquellos residuos generados durante el proceso de transformación, constituidos por restos de madera principalmente procedentes de la corteza de las coníferas. Este residuo presenta una alta densidad y calidad calorífica junto a un bajo grado de humedad, de esta forma, empleando un sistema de recogida bien organizado, estos residuos son utilizados para la generación de energía eléctrica en las 
propias instalaciones. La combustión se produce en la planta de biomasa, empleando la energía eléctrica producida para la propia actividad (producción de tableros) y para su venta a las compañías eléctricas (cogeneración). Tras el proceso de combustión citado, se generan cenizas cómo residuo, en concreto, en las instalaciones de la factoría de Tradema perteneciente al grupo Tafisa dedicada a la fabricación de tableros derivados de madera y ubicada en la ciudad de Linares, se generan entre 10 y $12 \mathrm{Tm}$ de residuos en forma de cenizas cada día, siendo el destino de éstas su acumulación en vertederos autorizados. Estas cenizas presentan un contenido en oxido de silicio del $52 \%$ y de óxido de calcio del $15 \%$.

Por otro lado, los silicatos de calcio son compuestos del sistema $\mathrm{CaO}-\mathrm{SiO}_{2}$ [6-7], entre los que destaca la wollastonita la cual se genera principalmente por proceso hidrotermales de sedimentos de calizas mezcladas con sílice. Otros compuestos de interés son el silicato dicálcico y el tricálcico que son fases importantes en el cemento. Estos silicatos tienen múltiples aplicaciones, entre estas destacan la producción de cerámicas aislantes térmicas, aditivos en la fabricación de polímeros, en la industria del cemento mejoran las propiedades de resistencia y durabilidad, en esmaltes reducen los puntos de fusión y otorgan mayor brillantez, en la fabricación de porcelanas dieléctricas, en la industria del acero cómo fundente o recubrimiento. Muy recientemente se ha encontrado un nuevo campo de aplicación como es el de los materiales cerámicos bioactivos; cómo biovidrios [8-10] y sustitución de huesos [11]

Con estos antecedentes, en el presente estudio se utilizan dichas cenizas cómo fuente de sílice y los residuos de mármol como fuente de óxido de calcio para la obtención de silicatos de calcio, estudiando la influencia de la presencia de humedad durante el mezclado inicial de los residuos. Además se estudian las propiedades tecnológicas de las probetas conformadas con dichos silicatos cálcicos para determinar su aplicación como material cerámico aislante térmico

\section{PARTE EXPERIMENTAL}

\subsection{Preparación de las muestras}

Las cenizas suministradas por la empresa Tradema se tamizaron con el fin de eliminar las partículas de gran tamaño y a continuación se introdujeron en un molino de bolas hasta conseguir un tamaño de grano de $100 \mu \mathrm{m}$. El residuo mármol en primer lugar se molturó en un molino de martillos y posteriormente al igual que las cenizas, se introdujo en el molino de bolas hasta que el tamaño de grano se redujo a 100 $\mu \mathrm{m}$. Ambos materiales, cenizas y mármol, se mezclaron con una relación molar 1:1 $\mathrm{CaO}-\mathrm{SiO}_{2^{\prime}}$ en dos etapas diferentes. En la primera etapa, el mezclado en fase seca, se realizó en el molino de bolas durante 2 horas y en la segunda etapa, el mezclado en fase húmeda, se realizó en agitador con $60 \%$ de humedad durante 2 horas a $90^{\circ} \mathrm{C}$. A continuación las mezclas pulverizadas obtenidas por las dos diferentes etapas, por mezclado en fases seca y fase húmeda de cenizas de biomasa y mármol, con objeto de obtener silicatos de calcio por reacción en estado sólido, se introdujeron en un horno y se sinterizaron a una temperatura de $1100^{\circ} \mathrm{C}$ durante 24 horas con una rampa de calentamiento de $20{ }^{\circ} \mathrm{C} / \mathrm{min}$. Para comprobar su aplicación como materiales de construcción aislantes térmicos de baja temperatura y estudiar las propiedades tecnológicas, el material pulverizado resultante se hidrató durante 20 minutos con $15 \%$ en peso de agua, para que tenga la suficiente plasticidad y ausencia de defectos, principalmente grietas, en la etapa posterior de moldeo por compresión. Para esta etapa se emplea una prensa hidraúlica de laboratorio Mega KCK-30 bajo cargas uniaxiales de $15 \mathrm{Tm}$. Las probetas conformadas tienen aproximadamente $60 \mathrm{~mm}$ de longitud, 30 $\mathrm{mm}$ de anchura y $10 \mathrm{~mm}$ de espesor. Después de conformar las piezas, se someten a una etapa de secado durante $24 \mathrm{~h}$ a $110^{\circ} \mathrm{C}$. Por último las probetas se sometieron a un proceso de curado, introduciendo las mismas en agua, 5 minutos al día, durante una semana, finalizando con una nueva etapa de secado durante 48 horas a $110^{\circ} \mathrm{C}$.

\subsection{Caracterización de las materias primas}

Los difractogramas de rayos-X de polvo para la determinación de las fases cristalinas presentes se realizaron empleando en un difractómetro automático Siemens D5000 con geometría $(\theta / 2 \theta)$ Bragg-Brentano usando radiación $\mathrm{K} \alpha_{1,2}$ equipado con un monocromador de grafito para eliminar la radiación $\mathrm{Cu} \mathrm{K} \beta$. La composición química de los residuos empleados se determina por fluorescencia de rayos-X (FRX) en un equipo Magix Pro (PW-2440).

Tanto las materia primas como los productos sintetizados, fueron analizados mediante análisis termogravimétrico (ATG) termodiferencial (ATD) empleando un equipo Mettler Toledo $850^{\mathrm{e}}$ donde las muestras de fueron puestas en el crisol de platino y calentadas a una velocidad de $20^{\circ} \mathrm{C} / \mathrm{min}$ desde temperatura ambiente hasta $1000{ }^{\circ} \mathrm{C}$. Los datos muestran los porcentajes de pérdida de peso en función de la temperatura y el diagrama ATD al mismo tiempo.

El contenido en carbonatos del residuo mármol fue determinado por el método del calcímetro de Bernard por la producción de $\mathrm{CO}_{2}$ después de la reacción del residuo con ácido clorhídrico diluido.

\subsection{Caracterización de las piezas conformadas.}

Con objeto de determinar si los productos fabricados cumplen con la legislación vigente para su utilización como materiales aislantes de construcción de baja temperatura, se han determinado sus propiedades físicas (densidad aparente, absorción de agua y succión de agua), mecánicas (resistencia a la compresión, resistencia a la heladicidad) y térmicas (conductividad térmica).

La densidad aparente se define como el cociente entre el peso del ladrillo seco y el volumen normalizado. Se realizaron medidas después de estar en la estufa a una temperatura de $110{ }^{\circ} \mathrm{C}$ durante 24 horas (seco). Se calcula como la ratio del peso seco del ladrillo conformado y su volumen estándar.

La capacidad de absorción de agua es una medida del humedecimiento cuando se sumerge un ladrillo totalmente en agua durante un periodo prolongado. El ensayo se realizó siguiendo la norma UNE 22-182.

Las probetas se desecan en estufa a $110{ }^{\circ} \mathrm{C}$, durante 24 horas. Una vez secas se realizan pesadas hasta que la diferencia de peso es inferior al 1\%, obteniendo un peso Gs. Después de enfriarlas al aire durante 24 horas, se introducen en agua lentamente hasta conseguir su inmersión completa. A las 24 horas de inicio de su inmersión se sacan del agua, secándose con un paño húmedo y se pesan, volviéndose a introducir en 
agua. Cada 24 horas se continúan pesando las muestras, hasta que en dos pesadas consecutivas se encuentren diferencias inferiores al $0.1 \%$, obteniendo un peso, Ge.

El agua absorbida es la diferencia entre las pesadas, Ge-Gs.

El porcentaje de absorción de cada probeta, se calcula mediante la aplicación de la expresión:

$$
A=((G e-G s) / G s) * 100
$$

La succión de agua de un ladrillo es la cantidad de agua absorbida durante una inmersión parcial de corta duración. Con este ensayo obtenemos la capacidad inicial de inhibición de agua por capilaridad. Para realizar este ensayo se sigue la norma UNE 67-031.

Las probetas se desecan en estufa a una temperatura comprendida entre 100 y $110{ }^{\circ} \mathrm{C}$ hasta que su peso sea constante, Pi. Se mide la superficie de la cara de la probeta que va a entrar en contacto con el agua. Se toma una bandeja y se llena con la cantidad de agua precisa para que, una vez colocada la pieza el nivel quede constante cubriéndola aproximadamente $3 \mathrm{~mm}$. Pasado un minuto se saca la probeta, se seca superficialmente con un paño y se obtiene su peso, Qi en gramos.

La succión de agua viene dada por la siguiente expresión:

$$
S=Q i-P i / A\left(\mathrm{~g} / \mathrm{cm}^{2} \cdot \mathrm{min}\right)
$$

Para la determinación experimental de la conductividad térmica de los ladrillos conformados se empleó el modelo de la casa térmica, para la cual se dispone de un dispositivo al efecto, con paredes y techo aislados. En una de las paredes se sitúa el material a ensayar. La transmisión de calor se debe a la diferencia de temperatura entre las zonas en las que se produce el intercambio y en nuestro caso la transmisión de calor se debe únicamente a la convección y conducción despreciando la transmisión de calor por radiación. En la casa térmica se enciende el wattímetro y una vez alcanzado el estado estacionario en el dispositivo se anotan los valores de temperatura de la cara interna y externa de la pared del material a ensayar, la del interior de la casa térmica y la exterior del laboratorio mediante termopares. El caudal de calor, $\mathrm{Q}$, en el interior de la casa térmica una vez alcanzado el estado estacionario puede ser evaluado por:

$$
Q=h_{i} A\left(T_{i}-T_{p i}\right)
$$

donde: $h_{i}$ es el coeficiente individual de transmisión de calor por convección aire-pared, en el interior del dispositivo; A: el área transversal a la transmisión de calor y $\mathrm{T}_{\mathrm{i}}$ y $\mathrm{T}_{\mathrm{p}}$ : las temperaturas del interior de la casa térmica y de la superficie interior de la pared respectivamente.

De la misma forma la transmisión de calor desde la superficie externa de la pared al aire se evalúa por una expresión análoga:

$$
Q=h_{e} A\left(T_{p e}-T\right)
$$

donde: $h_{e}$ es el coeficiente individual de transmisión de calor por el convección pared-aire, en el exterior del dispositivo; A: área transversal a la transmisión de calor; $\mathrm{T}_{\mathrm{pe}} \mathrm{y}$ $\mathrm{T}_{\mathrm{e}}$ : las temperaturas de la superficie exterior de la pared y del laboratorio respectivamente.
La transmisión de calor por conducción en la pared se calcula con la expresión:

$$
Q=k A\left(T_{i}-T_{p e}\right) / e
$$

donde: $\mathrm{k}$ es la conductividad térmica del material de la pared; e: el espesor de la pared.

Considerando que en el estado estacionario el caudal de calor es constante y que el valor del coeficiente de transmisión de calor por convección en el caso de habitaciones cerradas encontrado en bibliografía es $8,1 \mathrm{w} / \mathrm{m}^{2} \mathrm{~K}$, la combinación de las ecuaciones [3], [4] y [5] permiten calcular la conductividad térmica del material ensayado.

Para el ensayo de resistencia a la compresión se tomaron seis probetas de cada muestra. El ensayo de resistencia a compresión se realizó siguiendo la norma UNE 67-026, en una prensa de laboratorio Suzpecar CME 200 SDC.

Para iniciar el ensayo, previamente se deben colocar las superficies de apoyo de la máquina de ensayo, así como las caras de la probeta, y situarla en el centro de las placas de la máquina de ensayo.

Se mide el área A de las dos caras de apoyo y se realiza la media.

Todas las probetas fueron sometidas a un esfuerzo normal progresivamente creciente, aplicando la carga centrada en la cara superior de la probeta con una velocidad de aplicación no superior a los $20 \mathrm{MPa} / \mathrm{s}$ hasta la rotura.

La resistencia a la compresión de cada probeta se obtuvo dividiendo la carga máxima de la superficie media de sus dos caras de apoyo, siendo expresada en MPa con precisión de $0.1 \mathrm{MPa}$.

El efecto de la resistencia a la heladicidad se realiza de acuerdo con la normas UNE 67028, por medio de un sistema manual, sometiendo a las muestras a 25 ciclos de hielodeshielo (18 horas de hielo-8 horas de deshielo) utilizando un congelador, previa saturación de las probetas por inmersión en agua durante $48 \mathrm{~h}$.

La microestructura de las piezas conformadas se observó por microscopía electrónica de barrido (SEM) empleando un microscopio de transmisión electrónica de alta resolución JEOL SM 840. Las muestras fueron puestas en una rejilla de aluminio y cubiertas por una capa de oro usando un metalizador iónico JEOL JFC 1100. Se llevó a cabo también el EDAX para determinar la ratio de los constituyentes de las probetas analizadas.

\section{RESULTADOS Y DISCUSIÓN}

\subsection{Caracterización de las materias primas}

La composición química de los residuos, mármol y cenizas determinada por FRX se presenta en la Tabla 1. Los resultados indican que el residuo de mármol contiene un contenido significante de óxido de calcio (55.3\%). Además el mármol contiene cantidades menores de impurezas en forma de óxidos de magnesio y hierro, sílice y alúmina. La alta pérdida por ignición es atribuida a la pérdida del gas carbónico debido a la disociación parcial de la calcita y la dolomita. El residuo cenizas es una fuente potencial de óxidos. Su mayor componente es el $\mathrm{SiO}_{2}(52.53 \%)$, pero el $\mathrm{CaO}(15 \%)$ y otros óxidos como $\mathrm{Al}_{2} \mathrm{O}_{3^{\prime}} \mathrm{Fe}_{2} \mathrm{O}_{3^{\prime}}, \mathrm{MgO}, \mathrm{TiO}_{2^{\prime}} \mathrm{K}_{2} \mathrm{O}$ y $\mathrm{Na}_{2} \mathrm{O}$ que pueden 
actuar como mineralizadores están presentes en cantidades considerables. Los componentes minoritarios son el $\mathrm{MnO}$, $\mathrm{P}_{2} \mathrm{O}_{5}$ y $\mathrm{ZnO}$ que se encuentran en cantidades inferiores al 1 $\%$. La pérdida por ignición es de aproximadamente el $11 \%$ y puede ser atribuida a la pérdida de volátiles asociada a la descomposición de los diferentes compuestos, incluyendo el carbón total orgánico.

TABLA 1. COMPOSICIÓN QUÍMICA DE LOS RESIDUOS

\begin{tabular}{ccc}
\hline Óxidos constituyentes & Mármol & Cenizas \\
\hline $\mathrm{SiO}_{2}(\%)$ & $0.93 \pm 0.01$ & $52.53 \pm 0.01$ \\
$\mathrm{Al}_{2} \mathrm{O}_{3}(\%)$ & $0.36 \pm 0.01$ & $6.88 \pm 0.01$ \\
$\mathrm{Fe}_{2} \mathrm{O}_{3}(\%)$ & $0.16 \pm 0.01$ & $4.92 \pm 0.01$ \\
$\mathrm{MnO}(\%)$ & - & $0.10 \pm 0.01$ \\
$\mathrm{MgO}(\%)$ & $1.25 \pm 0.01$ & $2.62 \pm 0.01$ \\
$\mathrm{CaO}(\%)$ & $55.3 \pm 0.01$ & $14.95 \pm 0.01$ \\
$\mathrm{Na}_{2} \mathrm{O}(\%)$ & - & $1.19 \pm 0.01$ \\
$\mathrm{~K}_{2} \mathrm{O}(\%)$ & - & $1.96 \pm 0.01$ \\
$\mathrm{TiO}_{2}(\%)$ & - & $2.59 \pm 0.01$ \\
$\mathbf{P}_{2} \mathrm{O}_{5}(\%)$ & - & $0.62 \pm 0.01$ \\
$\mathbf{Z r}(\mathbf{p p m})$ & $7,05 \pm 1.0$ & $267.4 \pm 1.0$ \\
$\mathbf{L o I}(\%)$ & $42.0 \pm 0.01$ & $11.64 \pm 0.01$ \\
\hline
\end{tabular}
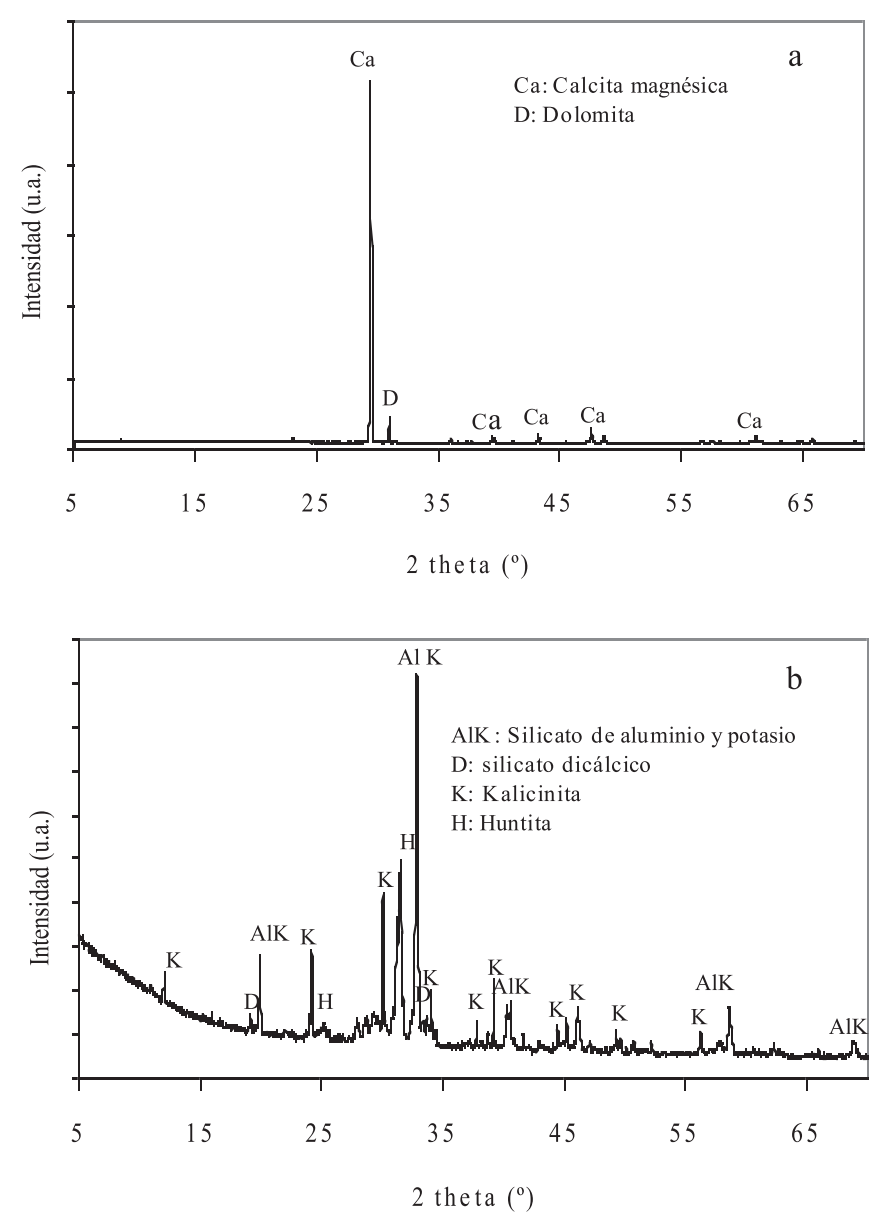

Figura 1. Difractograma de rayos-X de los residuos empleados como materia prima (a): mármol y (b) cenizas.
Los resultados de DRX y calcimetría de Bernard $\left(98 \% \mathrm{CaCO}_{3}\right)$ para el residuo mármol confirman que contiene una gran cantidad de calcita. El patrón de DRX de el residuo de mármol muestra, como se esperaba, un intenso pico correspondiente a la calcita magnésica $\left(\mathrm{Mg}_{0.03} \mathrm{Ca}_{0.97}\right)\left(\mathrm{CO}_{3}\right)$ y un pico más discreto que indica la presencia de dolomita $\left(\mathrm{Ca}\left(\mathrm{Mg}\left(\mathrm{CO}_{3}\right)_{2}\right)\right.$ observándose además trazas de cuarzo (Figura 1a).

El comportamiento térmico del residuo mármol se determinó por ATD-ATG y se presenta en la figura 2a. Se puede observar a $850{ }^{\circ} \mathrm{C}$ un fuerte pico endotérmico asociado a la descomposición del carbonato cálcico, $\mathrm{CaCO}_{3}$, en óxido de calcio y $\mathrm{CO}_{2}$ dando lugar a una pérdida de peso del $42 \%$ de acuerdo con los resultados obtenido por LOI. (Figura 2a).

El patrón de DRX del residuo cenizas se muestra en la Figura $1 b$, observándose los picos de difracción correspondientes a silicato de aluminio y potasio $\left(\mathrm{K}_{0.85} \mathrm{Al}_{0.85} \mathrm{Si}_{0.15} \mathrm{O}_{2}\right)$, silicato dicálcico $\left(\mathrm{Ca}_{2} \mathrm{SiO}_{4}\right)$, y minerales como kalicinita $\left(\mathrm{K}\left(\mathrm{HCO}_{3}\right)\right)$ y huntita $\left(\mathrm{Mg}_{3} \mathrm{Ca}\left(\mathrm{CO}_{3}\right)_{4}\right)$. El contenido de carbonatos en las cenizas es del $12,2 \%$.

La curva de ATD-ATG del residuo cenizas (Figura 2b) muestra un pico endotérmico que corresponde a la eliminación de la humedad presente en las cenizas y que se produce a temperaturas alrededor de los $150{ }^{\circ} \mathrm{C}$. También se producen dos picos exotérmicos correspondientes a los elementos inquemados presentes en las cenizas.


Figura 2. ATD-ATG de los residuos empleados como materia prima (a): mármol y (b) cenizas. 


\subsection{Caracterización de los materiales sintetizados}

Los datos de DRX de las dos muestras obtenidas tras mezclado de los residuos tanto en fase seca, como en fase húmeda y sinterización a $1100{ }^{\circ} \mathrm{C}$ se muestran en la Figura 3. En ambas rutas se forman silicatos como wollastonita $\left(\mathrm{CaSiO}_{3}\right)$; gelanita $\left(\mathrm{Ca}_{2} \mathrm{Al}(\mathrm{AlSi}) \mathrm{O}_{7}\right)$ y larnita $\left(\mathrm{Ca}_{2} \mathrm{SiO}_{4}\right)$, observándose también los picos de difracción de las materias primas que no han reaccionado completamente como calcita $\left(\mathrm{CaCO}_{3}\right)$, cuarzo $\left(\mathrm{SiO}_{2}\right)$ y portlandita $\left(\mathrm{Ca}(\mathrm{OH})_{2}\right)$. No obstante, en la síntesis por mezclado de reactivos en vía húmeda (ruta $\mathrm{RH}$ ) la calcita es la materia prima que menor reacciona mientras que en la síntesis por mezclado de residuos en vía seca ( ruta RSS) es el cuarzo el componente que reacciona en menor proporción.

El comportamiento térmico de los silicatos sintetizados por ambas rutas se presenta en la Figura 4. En ambos casos, se observa un pequeño pico endotérmico a $90{ }^{\circ} \mathrm{C}$ que se puede atribuir a la pérdida de humedad, dando una pérdida de masa del $0.3 \%$ y del $1.5 \%$ para la ruta seca y húmeda, respectivamente. A la temperatura de $490{ }^{\circ} \mathrm{C}$ se observa un fuerte endotérmico, mucho más intenso en la ruta en fase húmeda, correspondiente a la deshidratación (pérdida de agua de constitución) debido a la rotura del retículo cristalino de los silicatos formados. Finalmente a $790{ }^{\circ} \mathrm{C}$ se aprecia un fuerte endotérmico mucho más intenso en la síntesis por mezclado en fase seca debido a la descomposición térmica del $\mathrm{CaCO}_{3}$ (calcita) en $\mathrm{CaO}+\mathrm{CO}_{2}$ con una pérdida de masa del 5 $\%$ para la síntesis en fase seca y del $4 \%$ para la síntesis en fase húmeda.

\subsection{Caracterización de las probetas conformadas}

Con objeto de determinar si los materiales sintetizados podían emplearse como cerámicos aislantes térmicos de baja temperatura, se procedió a la compresión de los mismos empleando cargas uniaxiales de $15 \mathrm{Tm}$ para elaborar ladrillos con una sección transversal de 30 x $10 \mathrm{~mm}$ y una longitud de $60 \mathrm{~mm}$. Se ha investigado el efecto de la densidad aparente, absorción y succión de agua del producto final, resistencia a la compresión y conductividad térmica.

Los datos de densidad aparente de las probetas sinterizadas con los productos procedentes de las dos rutas de síntesis son similares (Tabla 2), obteniendo un valor en torno a los $2 \mathrm{~g} / \mathrm{cm}^{3}$. Estos datos indican que la porosidad total de las probetas es prácticamente la misma.

La porosidad total de las probetas puede ser de dos tipos, abierta y cerrada. La porosidad abierta puede determinarse a partir de los valores de absorción de agua y para la porosidad cerrada se debe determinar la densidad real de las piezas. Cuanto menor es la absorción de agua se espera que estos tengan una mayor durabilidad y más resistencia al medio.

Los datos de adsorción de agua (Tabla 2) indican que las probetas sintetizadas con los residuos mezclados por vía húmeda presentan un mayor valor de absorción de agua $(12.7 \%)$, mientras que las probetas obtenidas con los residuos mezclados por vía seca alcanzan un porcentaje de absorción de agua del $9.0 \%$. Estos datos indican que cuando se realiza la síntesis por mezclado en fase húmeda se consigue un material con mayor porosidad abierta.

Se ha determinado la succión de agua de las probetas (Tabla 2), obteniendo, como cabría esperar de acuerdo con los datos de absorción de agua, un valor de succión tres

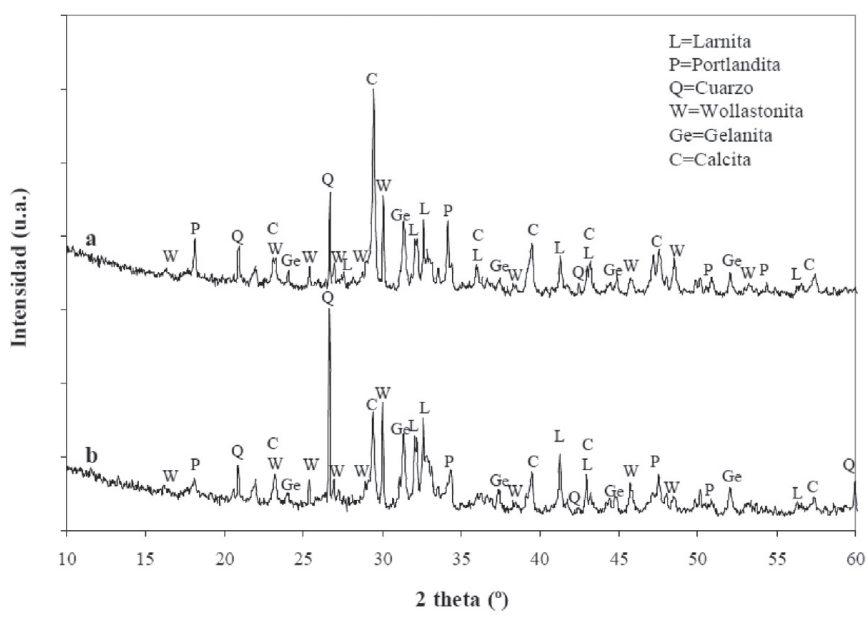

Figura 3. Difractogramas de rayos- $X$ de los silicatos cálcicos sintetizados (a): mezcla de reactivos en fase húmeda y (b) mezcla de reactivos en fase seca.
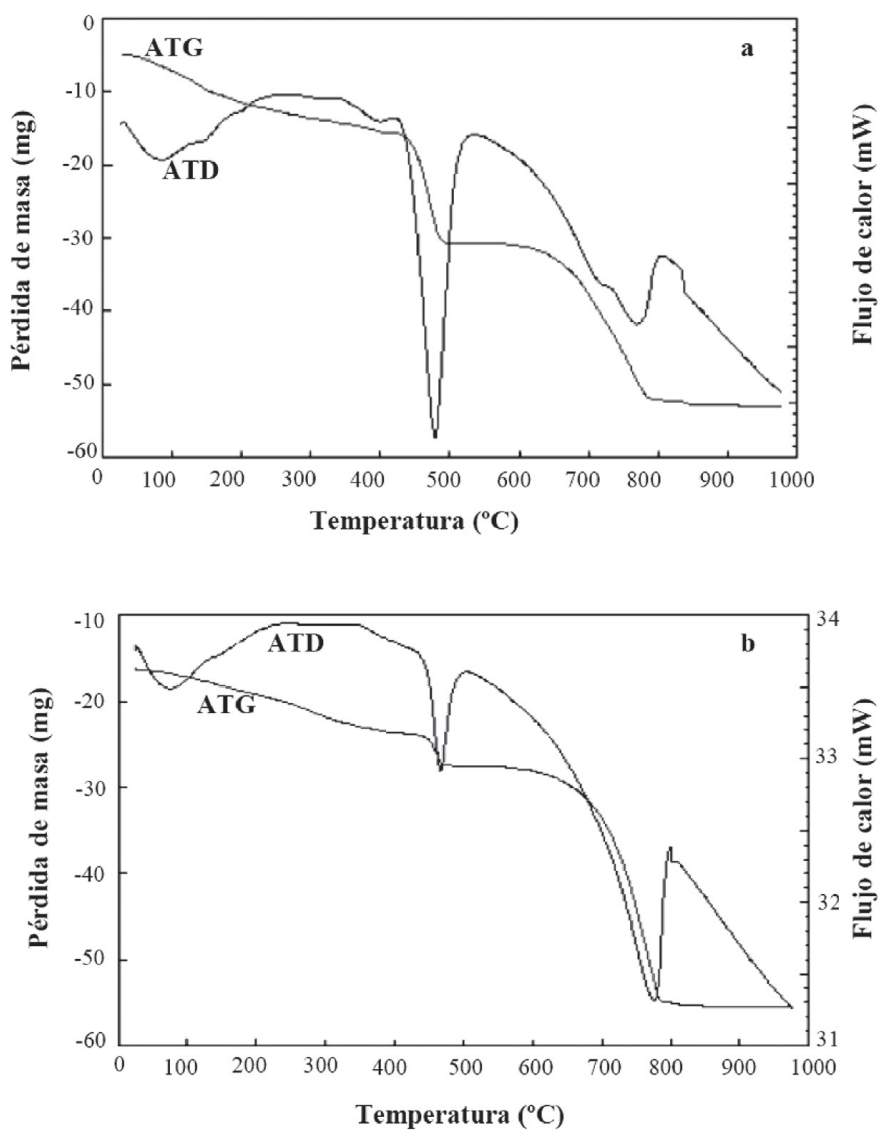

Figura 4. ATD-ATG de los silicatos cálcicos sintetizados (a): mezcla de reactivos en fase húmeda y (b) mezcla de reactivos en fase seca. 
veces superior cuando la síntesis se realiza con los residuos en fase húmeda $\left(0.008 \mathrm{~g} / \mathrm{cm}^{2} \mathrm{~min}\right)$. De nuevo estas probetas presentan una mayor porosidad superficial interconectada. Esta propiedad afecta de forma significativa a la calidad del material final y a su durabilidad, por lo que se deben evitar valores elevados de esta propiedad que podrían provocar piezas con defectos y de menor durabilidad. De acuerdo con la norma UNE-67019 la succión de agua debe ser inferior a $0.45 \mathrm{~g} / \mathrm{cm}^{2} \mathrm{~min}$.

La resistencia a la compresión en materiales cerámicos es el índice de calidad ingenieril más importante para materiales de construcción, dado que estos materiales ejercen funciones estructurales en el edificio. La resistencia a compresión de los ladrillos según normas UNE-67.046-88 debe ser de al menos $10 \mathrm{MPa}$.

La resistencia a compresión (Tabla 2) es menor para las probetas sintetizadas a partir del mezclado de residuos por fase húmeda, debido posiblemente a que este material presenta una mayor porosidad abierta e interconectada superficial como indican los datos de absorción y succión de agua, respectivamente. Esta porosidad puede actuar como centro de acumulación de tensiones permitiendo la propagación de las grietas. No obstante, en ambos casos se consiguen valores de resistencia a compresión muy superiores a los permitidos.

TABLA 2. PROPIEDADES DE LOS LADRILlOS CONFORMADOS CON LOS SILICATOS CÁLCICOS SINTETIZADOS

\begin{tabular}{lcc}
\hline & \multicolumn{2}{c}{ Muestra } \\
\cline { 2 - 3 } Propiedad & Fase Seca & $\begin{array}{c}\text { Fase } \\
\text { Húmeda }\end{array}$ \\
\hline Densidad aparente $\left(\mathrm{g} / \mathrm{cm}^{3}\right)$ & $2.05 \pm 0.035$ & $1.93 \pm 0.034$ \\
Adsorción $(\%)$ & $9.03 \pm 3.083$ & $12.67 \pm 0.209$ \\
$\begin{array}{l}\text { Succión }\left(\mathrm{g} / \mathrm{cm}^{2} \text { min) }\right. \\
\text { Resistencia a compresión } \\
\text { (MPa) }\end{array}$ & $0.002 \pm 0.001$ & $0.008 \pm 0.003$ \\
$\begin{array}{l}\text { Conductividad térmica } \\
\text { M }\end{array}$ & $59.3 \pm 2.564$ & $48.3 \pm 2.324$ \\
\hline
\end{tabular}

Con objeto de determinar la aplicabilidad de los materiales conformados como cerámico aislante térmico se procedió a determinar la conductividad térmica. Esta propiedad no varía mucho obteniendo un valor de $0.18 \mathrm{~W} / \mathrm{mK}$ para las probetas sintetizadas por mezclado en vía seca y $0.12 \mathrm{~W} / \mathrm{mK}$ para las probetas sintetizadas por mezclado en vía húmeda. Se puede ver que el valor de conductividad térmica es ligeramente menor para las probetas sintetizadas por vía húmeda $(\mathrm{RH})$, como cabría de esperar, ya que presentan una mayor porosidad total, como indican los datos de densidad aparente.

De acuerdo con Avgustinik [13], es la porosidad cerrada la causa de aislamiento térmico de los materiales. Así pues hay que estudiar la influencia de la cantidad, calidad y distribución de la porosidad en el comportamiento térmico de los materiales. Los poros grandes son los que originan un aumento de la conductividad térmica, mientras que los más finos y uniformes le dan mayor capacidad de aislamiento térmico.
Puesto que las probetas sintetizadas por vía húmeda presentan mayor porosidad abierta e interconectada superficial como indican los datos de absorción y succión de agua, respectivamente, y por tanto menor cantidad de porosidad cerrada, estas muestras deben contener poros más finos y uniformes que la muestra sintetizada por mezclado de reactivos en fase seca, que a pesar de tener mayor porosidad cerrada su conductividad térmica es mayor indicando que los poros de esta muestra son grandes y por tanto originan una disminución de su capacidad de aislamiento.

El análisis morfológico de las probetas sintetizadas se ha determinado mediante microscopía de barrido (SEM) acoplada a EDAX para determinar la ratio de los elementos constituyentes.

En ambas micrografías se observa la presencia de $\mathrm{CaCO}_{3}$ y pequeñas cantidades de cuarzo, ambos sin reaccionar con la formación de partículas de silicatos cálcicos, en las que el $\mathrm{CaO}$ y el $\mathrm{SiO}_{2}$ son los componentes mayoritarios, aunque también contienen $\mathrm{Al}_{2} \mathrm{O}_{3}, \mathrm{MgO}, \mathrm{NaO}$, teniendo la muestra obtenida por mezclado en vía húmeda además $\mathrm{Fe}_{2} \mathrm{O}_{3^{\prime}}$ mientras que en la obtenida por mezclado por vía seca se observan trazas de $\mathrm{TiO}_{2}$, como muestra el análisis EDAX (Figura 5). En ambas probetas se observa además porosidad, si bien la presencia de porosidad abierta es superior en las probetas sintetizadas por mezclado de los reactivos en fase húmeda como indican los datos de absorción de agua. Además en ambas muestras se observa la presencia de porosidad cerrada, que será la encargada de aumentar el aislamiento térmico del material, aunque el tamaño del poro es distinto, presentando un mayor tamaño de poro las muestras obtenidas por reacción entre cenizas de biomasa y mármol mezcladas en fase seca.

Finalmente, las probetas fueron sometidas al ensayo de heladicidad, que es un índice de susceptibilidad a ciclos sucesivos de heladas y deshielos. Se define por la disminución de la resistencia a la compresión de las probetas antes y después de haber sido sometidas a 25 ciclos de hielo y deshielo. Completados los 25 ciclos, se procedió a la inspección ocular de las piezas, y se comprobó que durante el ensayo no se produjeron roturas de las probetas, ni tampoco deterioro superficial como exfoliaciones, fisuras o desconchados como se puede ver en las fotografías adjuntas (Figura 6). Una vez finalizados los ciclos de hielo-deshielo las probetas se sometieron de nuevo al ensayo de compresión, comprobándose que mientras la resistencia a la compresión en las probetas sintetizadas por mezclado en fase seca, incluso incrementa, no obstante, en las probetas sintetizadas por mezclado en fase húmeda, disminuye un $57.5 \%$ (Figura 7).

Estos resultados podrían explicarse debido a que las tensiones originadas en el cuerpo de las probetas, a causa del incremento de volumen del agua al congelarse, aproximadamente del $9 \%$, (Quijano, 2007) [14] y viceversa, quedan contrarrestadas, ya que durante el proceso de congelación el agua se desplaza por la formación de hielo en la pasta y se acomoda de tal forma que no resulta perjudicial; las burbujas de aire en la pasta suministran cámaras donde se introduce el agua y así se alivia la presión hidráulica generada, protegiendo al producto contra daños por congelación y descongelación.

Por tanto, el incremento observado en la resistencia a compresión de las probetas sintetizadas por mezclado en fase seca tras la etapa de hielo-deshielo podría ser debida a que estas muestras presentan una menor proporción de porosidad 

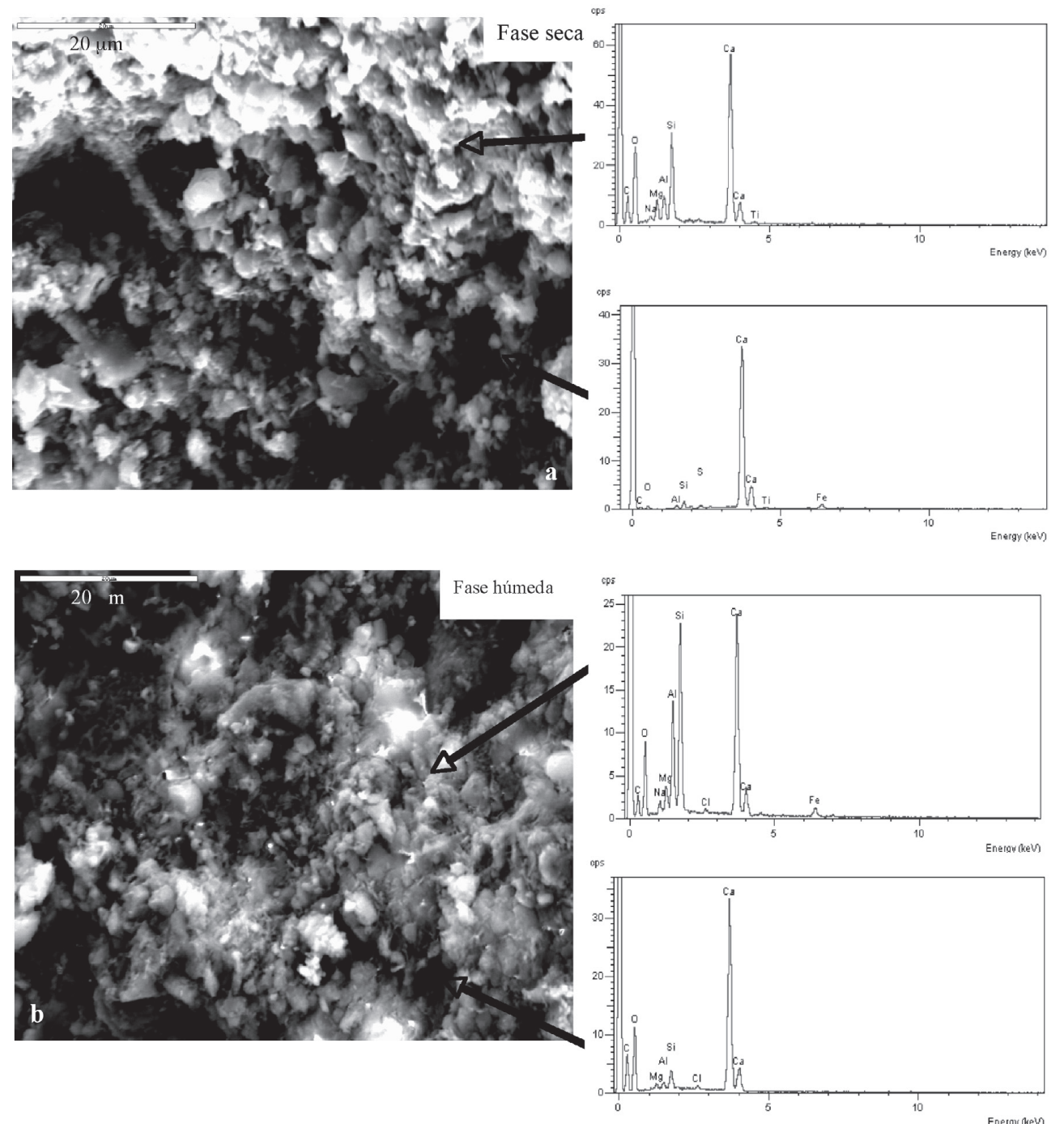

Figura 5. Micrografías SEM y análisis EDAX de los silicatos cálcicos sintetizados (a) mezclado de residuos en fase seca y (b) mezclado de residuos en fase húmeda
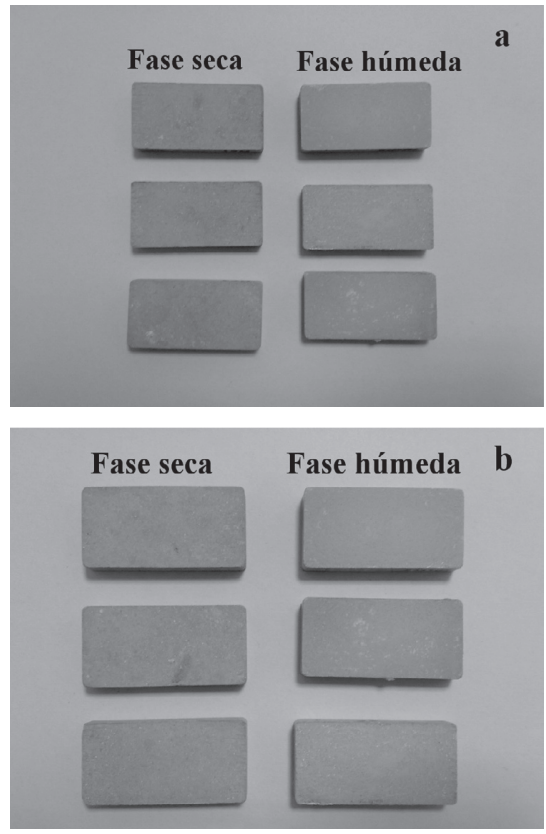

Figura 6. Probetas de silicatos cálcicos (a) antes y (b) después del ensayo de heladicidad

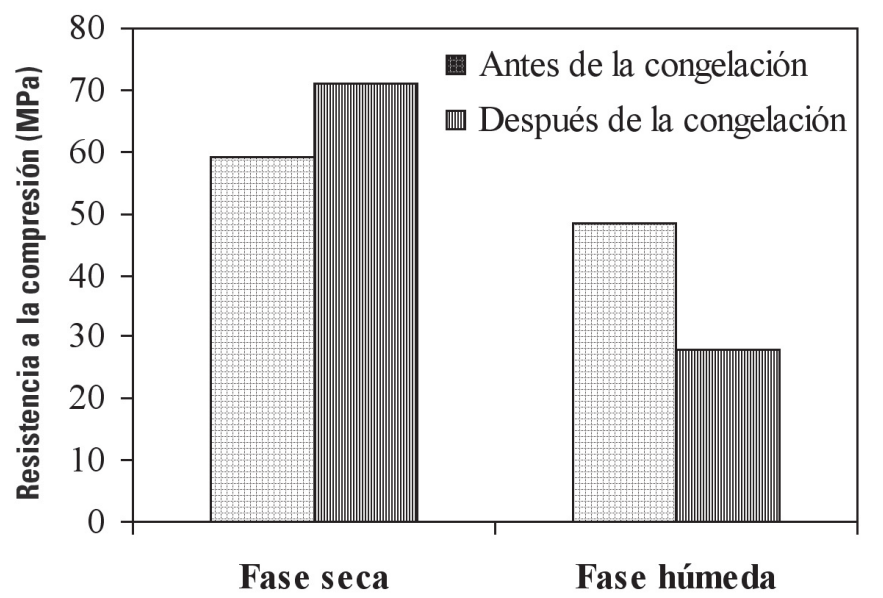

Figura 7. Resistencia a la compresión antes y después del ensayo de heladicidad 
abierta e interconectada, pudiendo actuar los poros cerrados como cámaras donde se introduce el agua y se alivia la presión hidráulica protegiéndolas durante este proceso.

\section{CONCLUSIONES}

A partir de los residuos, cenizas cómo fuente de sílice, y mármol como fuente de óxido de calcio en reacción molar $\mathrm{CaO}: \mathrm{SiO}_{2} 1: 1$ se consigue obtener silicatos de cálcio a $1100{ }^{\circ} \mathrm{C}$ de forma económica. No obstante, queda aún materia prima sin reaccionar. En la ruta en estado sólido el contenido de cuarzo sin reaccionar es mayor que el de calcita, mientras que en la ruta húmeda ocurre lo contrario como indican los resultados de DRX.

Los materiales sintetizados por ambas rutas fueron conformados para comprobar su uso como aislante cerámico térmico de baja temperatura, ambos materiales presentan valores bajos de conductividad térmica, consiguiendo mayor capacidad de aislamiento con el material sintetizado por la ruta húmeda, ya que presenta una mayor porosidad total, como indican los datos de densidad aparente. No obstante, el material sinterizado por mezclado de reactivos en fase seca presenta mejores propiedades tecnológicas, y al presentar también un bajo valor de conductividad térmica, $0.18 \mathrm{w} / \mathrm{m}^{2} \mathrm{~K}$, es preferible para su uso como material cerámico aislante.

\section{BIBLIOGRAFÍA}

1. J.M.F. Ferreira, P.J. Guedes, P. Torres, R.S. Manjate, H.R. Fernandes, in: Pecchio, et al. Editors. "Recycling of Industrial Wastes-an Overview about Succesful Case Studies". Applied Mineralogy, ICAM-BR São Paulo p.33 (2004)

2. A. Gorokhovsky, V. Gorokhovsky, and D. Mescheryakov. "Glass-ceramic materials based on complex utilization of industrial wastes: Structural features". Glastech. Ber. Glass Sci. Technol., 73[1] 374-377 (2000).

3. M. Dondi, M. Marsigli, B. Fabri. "Recycling of urban and industrial wastes in brick production: a review". Tile \& Brick Int. 13 [4] 302-315 (1997).

4. M. Dondi, M. Marsigli, B. Fabbri. "Recycling of industrial and urban wastes in brick production: a review". Tile Brick Int. 13 218-225 (1997).

5. R. Caligares, N. Quaranta, M. Caligares, E. Benavidez. “Materias primas no tradicionales en la industria cerámica". Bol. Soc. Esp. Ceram. Vidrio 39 623-626 (2000)

6. E.M. Levin, C.R. Robbins, H.F. HcMurdie. Phase Diagrams for Ceramists, Amer. Ceram. Soc., 1996, Fig 237.

7. J.R. Taylor, A.T. Didsdale. “Thermodinamic and phase diagram data for the CaO-SiO system. Calphad 14 71-88 (1990).

8. J H.G. Kurczyk, J. Wuhrer."Synthetic wollastonite and its use in ceramic bodies". J. Soc. Chem. 31 1-6 (1979)

9. P.N. de Aza, A.H. de Aza, S. de Aza. "Cristalline Bioceramic Materials". Bol. Soc. Esp. Ceram. Vidrio 44[3] 135-145 (2005)

10. P.N. de Aza, A.H. de Aza, P. Pena, S. de Aza. "Bioctive glasses and glassceramics". Bol. Soc. Esp. Ceram. Vidrio 46[2] 45-55 (2007).

11. L.L. Hench. "Bioceramic from concept to clinic", J. Am. Ceram. Soc. 74 (4) 1487-1510, (1991).

12. L.L. Long, L.D. Chen, J. Chang. "Low temperature fabrication and characterization of $\beta-\mathrm{CaSiO}_{3}$ ceramics". Ceram. Int. 33 (4) 457-460 (2006).

13. A.l. Avgustinik, Cerámica. Ed. Reverté. Barcelona.

14. S. Quijano. 2007. Universidad de Colima. Facultad Ciencias Marinas. www. Ciam.ucol.mx/directorios/1327/ clases/geología6.ppt. 